\title{
La dynamique lexicale en Droit civil français : un regard sur la terminologie des institutions célébrant et enregistrant les mariages officiels à travers les siècles
}

\author{
Beatriz Curti-Contessoto ${ }^{1, *}$, Isabelle Oliveira ${ }^{2}$, et Lidia Barros $^{3}$ \\ ${ }^{1}$ Université de São Paulo (USP), São Paulo, Brasil \\ ${ }^{2}$ Université Sorbonne Nouvelle - Paris 3, Paris, França \\ ${ }^{3}$ Université de l'Etat de São Paulo (UNESP), São José do Rio Preto, Brasil
}

Résumé. Cet article s'intéresse à l'évolution de la terminologie désignant les endroits où les mariages officiels français ont été célébrés et enregistrés au cours des XVIe, XVIIe, XVIIIe, XIXe, XXe et XXIe siècles. Nous présenterons, tout d'abord, les concepts de terminologie, langage de spécialité, terme, néologie et nécrologie. Nous entamerons également une discussion autour de quelques questions liées aux aspects socioculturels qui peuvent influencer l'évolution des langues, en particulier, des langages de spécialité. Ensuite, nous exposerons la méthodologie adoptée dans notre étude sous une perspective diachronique. Puis, nous analysons l'évolution de la terminologie en question et nous ferons un lien entre elle et les aspects socioculturels et historiques de la France.

\begin{abstract}
The lexical dynamics in Civil Law: a look at the terminology of institutions celebrating and registering official marriages in France throughout the centuries. This article focuses on the terminology designating the places where French official marriages were celebrated and registered throughout the sixteenth, seventeenth, eighteenth, nineteenth, twentieth and twenty-first centuries. First, the concepts of terminology, special language, term, neology and necrology are presented. Some questions related to the socio-cultural aspects that can influence the evolution of languages and special languages, are also discussed. Then, the methodology adopted in this study from a diachronic perspective is exposed. After doing this, the evolution of this terminology are analyzed, relating it to socio-cultural and historical aspects of France.
\end{abstract}

\section{Introduction}

Au cours du XVIe siècle, la France a connu une période de conflit entre les protestants et l'Eglise catholique à cause de nouvelles idées répandues par le protestantisme. En réponse à cela, l'Eglise a convoqué le Concile de Trente, qui a déterminé, par le Décret

\footnotetext{
*Auteure correspondante : bfcurti@gmail.com
} 
Tametsi (1562-1563), le caractère obligatoire de la célébration canonique des mariages contractés par des croyants et la compétence exclusive de l'Eglise dans les affaires matrimoniaux (Simonis \& Simonis 2001). Ainsi, seuls les catholiques pouvaient faire reconnaître légalement leurs mariages à cette époque-là.

C'était seulement à la fin du XVIIIe siècle que ce scénario a changé. En 1787, le Roi français a reconnu que le mariage entre non-catholiques pouvait être officiel après avoir été enregistré dans une paroisse par un prêtre catholique ou dans une maison royale par un juge du Royaume. Dès lors, tous les citoyens français pouvaient se marier légalement.

Quelques années plus tard, la Révolution française (1789-1799) a eu lieu. Elle défendait fortement le principe de laïcisation de l'Etat. Pendant cette Révolution, la République française a été instituée - ce qui a permis la séparation entre l'institution religieuse et l'autorité publique (Dalloz \& Dalloz 1845). Parmi les conséquences de cet évènement, qui a changé le cours de l'histoire de cette nation, nous soulignons l'institution du mariage civil au moyen de la Constitution du 3 septembre 1791 (France 1791). Ainsi, le mariage officiel avait perdu son caractère religieux. A cause de cela, les paroisses catholiques n'étaient plus les entités responsables de la célébration et de l'enregistrement des mariages officiels, c'està-dire les unions qui étaient valables aux yeux de l'Etat et de la société. Il a ensuite fallu créer d'autres institutions qui devaient s'occuper de cette question selon l'organisation politique et administrative de la République.

En l'occurrence, nous considérons dans notre analyse que la Révolution française a été l'un de grands facteurs qui a influencé la terminologie du domaine juridique français, en particulier, celle du domaine du Droit civil. D'ailleurs, d'autres aspects socioculturels ont influencé cet ensemble terminologique. De cette façon, nous nous proposons d'étudier le rapport entre ces aspects et la dynamique de la production lexicale des termes désignant les institutions responsables de la célébration et de l'enregistrement des mariages officiels de 1539 , où a été publiée la première loi abordant ce sujet, jusqu'en 2017, qui fait référence à l'année de la loi la plus récente en ce qui concerne les règles de célébration et d'enregistrement des mariages civils français.

Par rapport au cadre théorique adopté dans cette analyse, nous nous sommes axées surtout sur des recherches dans une perspective diachronique en Terminologie. Pour la vérification des questions socioculturelles intrinsèques à la terminologie étudiée, nous nous sommes basées sur une bibliographie spécialisée en Histoire de France et en Droit français. En guise de conclusion, nous présenterons, dans cet article, les termes étudiés et leurs contextes d'usage trouvés dans notre corpora (le LFCorpus et l'AMFCorpus), qui sont composés respectivement des lois concernant la thématique étudiée et des actes de mariage français délivrés entre les XVIe et XXIe siècles.

\section{Le cadre théorique adopté}

Dans cette étude, nous considérons que le terme terminologie est constitué d'un double sens car « il peut signifier les termes [...] représentant l'ensemble d'unités lexicales typiques d'un domaine [de spécialité] ou le champ d'études de cet ensemble » (Krieger et Finatto 2004, 13, notre traduction). Afin de les différencier, nous déterminons que, dans cet article, la notion de champ d'études des terminologies est écrite en $T$ majuscule, et que la notion d'ensemble des termes au moyen desquels les textes des domaines de spécialités expriment leurs concepts est orthografiée en $t$ minuscule.

Le champ d'études de la Terminologie, ce sont les langages de spécialité, utilisés par une communauté de spécialistes dans un domaine particulier à des fins de communication (Pavel \& Nolet 2003). Les langages de spécialité ont un lexique spécialisé (les terminologies) au moyen duquel un domaine du savoir transmet ses connaissances. L'unité terminologique est donc considérée une unité lexicale qui désigne un contenu spécifique dans un domaine spécialisé ${ }^{1}$. 
En Terminologie, les termes peuvent être étudiés selon différentes lignes théoriques. Dans notre étude, nous nous basons sur des approches qui explorent la diachronie, à savoir la Socioterminologie, la Théorie Communicative de la Terminologie (TCT) et la Terminologie Diachronique. Elles comprennent l'existence de variations lexicales et conceptuelles des langages de spécialité, ainsi que les aspects socioculturels et historiques qui y sont intrinsèques.

La Socioterminologie et la TCT sont deux lignes théoriques qui sont relativement proches l'une de l'autre car elles prennent en compte la dimension textuelle et discursive des termes et étudient la variation terminologique du point de vue dénominatif et sémantique. Pourtant, il y a des différences théoriques entre ces deux approches.

D'un côté, pour la Socioterminologie, " les termes servent à transmettre les significations qui sont socialement réglementées et ancrées dans les pratiques institutionnelles ou dans la connaissance » (Alves 2003, 229, notre traduction). Des sujets liés au caractère dynamique des termes, comme leurs usages sociaux, leur apparition, leur circulation et leur implantation, par exemple, sont donc étudiés (Gaudin 2005).

De l'autre, la TCT comprend que les termes « sont des unités linguistiques qui doivent être considérées dans une perspective polyédrique, c'est-à-dire dans leurs aspects linguistiques, cognitifs et sociaux » (Cabré 1998, 123, notre traduction). Ainsi, ce qui donne à l'unité lexicale le statut de terme, ce sont les conditions pragmatiques qui l'adaptent à un type de communication particulier (Cabré 1999).

A la lumière de ces considérations, le terme peut être conçu comme une unité qui, dans un système structuré, synthétise le concept d'un point de vue linguistique (auquel nous avons accès par l'analyse de ses comportements discursifs et sa morphosyntaxe) (Conceição 1999). En ce sens, le terme peut également être considéré une unité du système de connaissances d'un domaine et une unité représentative de ce système (Conceição 1999). Une fois qu'il est constitué d'une désignation qui renvoie à un concept, nous considèrons également que sa désignation est de l'ordre de la langue et que son concept est de l'ordre de la pensée (Depecker 2002, 112).

D'ailleurs, le terme est une unité lexicale spécialisée de la langue et, à cause de cela, il est soumis aux interférences et influences diverses (Curti-Contessoto 2018). De cette façon, cette étude suppose que, puisque le lexique des langues en général est dynamique car il change et s'enrichit constamment, les langages de spécialité, en tant que sous-systèmes linguistiques, n'échappent pas à ce processus (Barros 1998). Cette dynamique de production lexicale résulte principalement « d'un besoin de nomination ou d'un fait social qui, à un moment de l'histoire de la société, détermine la création d'une nouvelle unité lexicale » (Alves 2009, 1821). À titre d'exemple, nous citons la Révolution industrielle grâce à laquelle des changements sociaux, économiques et politiques ont eu lieu. A son tour, ces transformations ont eu des répercussions sur le niveau du vocabulaire. Ainsi, pour nommer chaque nouvelle invention, chaque nouvelle situation ou activité, produit, service, revendication, loi, etc., de nouveaux termes correspondants étaient créés (Barros 2004).

En ce sens, nous comprenons que la Révolution française et l'institution de la République dans ce pays ont joué un rôle similaire à celui de la Révolution industrielle en ce qui concerne la création de nouveaux termes spécifiquement dans le domaine du Droit français. De cette façon, en raison de la restructuration de l'Etat après l'institution de la République en France, une réorganisation juridique a été faite, et la mise en place d'une terminologie reflétant les idéaux révolutionnaires était nécessaire. Ainsi, des lois ont été établies, de nouvelles institutions publiques ont été créées, des fonctions publiques ont été déléguées et de nouveaux services publics ont été attribués. Plus précisément, le mariage civil a été institué en 1791. Il est devenu la seule union officielle reconnue par l'Etat républicain et n'était plus lié à l'Eglise catholique. Conséquemment, des termes ont été créés pour nommer les lieux où ce nouveau type de mariage devrait être célébré et enregistré selon les principes républicains à partir de ce moment-là. 
Fondées sur les approches théoriques présentées, nous comprenons que les termes doivent être étudiés dans leur contexte communicationnel et que les aspects historiques et temporels qui y sont intrinsèques doivent être analysés afin de vérifier la variation terminologique dans l'espace et le temps. Ce phénomène se manifeste principalement à deux niveaux, à savoir l'expression et le concept. Dans cet article, nous nous intéressons surtout à celui qui fait référence à la variation dénominative (ou lexicale).

Ce type de variation peut être conçu comme le phénomène qui se manifeste quand un même concept est désigné par des termes différents (Freixa 2006). Ces variantes peuvent exister à cause des raisons diverses, parmi lesquelles nous soulignons la variation chronologique qui est, selon Freixa (2014), un sous-type de la cause dialectale.

Il existe deux processus liés à cette variation, à savoir l'apparition et la disparition de termes véhiculés dans un domaine de spécialité. Dans notre étude, nous considérons qu'en observant ces processus, nous pouvons identifier la variation dans l'espace et le temps.

L'émergence de nouveaux ensembles terminologiques est liée au processus de création lexicale appelé néologie (Alves 2007). La néologie terminologique fait référence à ce qui est nouveau (la « nouveauté ») dans un domaine de spécialité en particulier (Picton 2014). En ce sens, les nouveaux termes qui apparaissent dans le contexte communicationnel d'un domaine sont appelés termes néologiques. Ces termes peuvent se stabiliser et continuer à être utilisés ou peuvent ne plus être véhiculés après un certain temps. Cela arrive lorsque leur utilisation ne devient pas stable dans le domaine où ils sont apparus.

A son tour, la disparition des termes peut être appelée nécrologie. Contrairement à la néologie, il s'agit de ce qui est devenu obsolète ( «ancien ») et est tombé en désuétude dans un domaine de spéciliaté (Picton 2014).

La néologie et la nécrologie peuvent être liées l'une à l'autre. En ce sens, un terme néologique peut émerger pour désigner un nouveau concept qui remplace un concept déjà existant qui, à son tour, devient obsolète - ce qui rend nécrologique le terme désignant le concept remplacé. Ce terme néologique peut ne pas devenir stable et, dans ce cas, il disparaît du lexique du domaine de spécialité. Ce néologisme devient donc un nécrologisme (Dury et Drouin 2009).

Cependant, ce rapport n'existe pas toujours. En ce sens, un terme néologique peut être créé pour nommer un concept totalement nouveau dans le domaine sans remplacer une expression déjà existante. De plus, l'usage d'un terme peut être abandonné à cause d'autres raisons telles que « la disparition d'un terme [sans lien avec la création d'une autre unité terminologique], la disparition d'une partie d'un terme, le changement d'un statut grammatical et / ou la disparition d'un sens pendant une période donnée » (Dury et Drouin 2009, 19).

Toutes ces questions sont explorées par la Terminologie Diachronique à partir de laquelle notre travail se fonde également. Cette approche théorique considère que, outre les phénomènes de réduction et d'expansion qui arrivent aux termes existants, les unités terminologiques, ainsi que les concepts qu'elles désignent, apparaissent et disparaissent sous l'influence des changements socioculturels et historiques d'un peuple. Pour cette raison, d'une part, certains termes et concepts deviennent obsolètes et tombent donc en désuétude ; d'autre part, de nouvelles terminologies et concepts qui accompagnent « l'évolution » de la société sont créés au fil du temps.

Dans cet article, nous proposons d'analyser l'évolution lexicale des termes qui désignent les institutions dans lesquelles les mariages officiels ont été célébrés et enregistrés entre les années 1539 et 2017. En ce sens, nous avons l'objectif d'étudier la dynamicité de la production lexicale de cet ensemble terminologique et de faire un rapport entre elle et les aspects socioculturels, politiques et historiques de la France au cours de cette période. Dans le point suivant, nous présentons la méthodologie adoptée pour poursuivre cet objectif. 


\section{Méthodologie}

Pour mener cette recherche, deux corpora dynamiques ont été créés. L'un s'appelle LFCorpus et se compose de 41 lois, décrets et amendements portant sur les règles du mariage officiellement reconnu par l'Etat depuis la première disposition sur cette matière (1539) jusqu'au dernier changement législatif en ce qui concerne les lieux où les mariages doivent être célébrés et enregistrés en France (2017). Chacun de ces documents juridiques représente un sous-corpus statique du LFCorpus et a été nommé par le code LF[ANNEE] pour indiquer l'année de publication de la loi à laquelle il fait référence.

L'autre corpus que nous avons créé s'appelle AMFCorpus. Il rassemble 102 actes de mariage français qui ont été délivrés entre les XVIe et XXIe siècles. Ainsi, leurs dates de délivrance comprennent la même période de publication des documents juridiques composant le LFCorpus. Cela signifie que les mariages enregistrés dans ces actes ont été célébrés à la lumière des principes juridiques français en vigueur entre 1539 et 2017.

L'AMFCorpus est aussi composé des sous-corpora statiques qui correspondent à chaque acte de mariage auquel nous avons eu accès et qui ont été nommés par le code $\mathrm{AMF}[\mathrm{ANNEE}]$. Une fois que ces documents ${ }^{2}$ ont été mis à notre disposition au format image, nous avons dû taper le contenu de tous ces actes pour que nous puissions les sauvegarder au format txt.

Après avoir compilé les corpora mentionnés, tous les deux ont été stockés dans le programme de traitement de données textuelles et lexicales appelé Hyperbase $e^{3}$ pour que nous puissions y identifier la terminologie en question. Sur ce logiciel, nous avons généré des listes de concordance pour chaque corpus en étude. A titre d'illustration, nous présentons un exemple de cette étape de notre recherche:

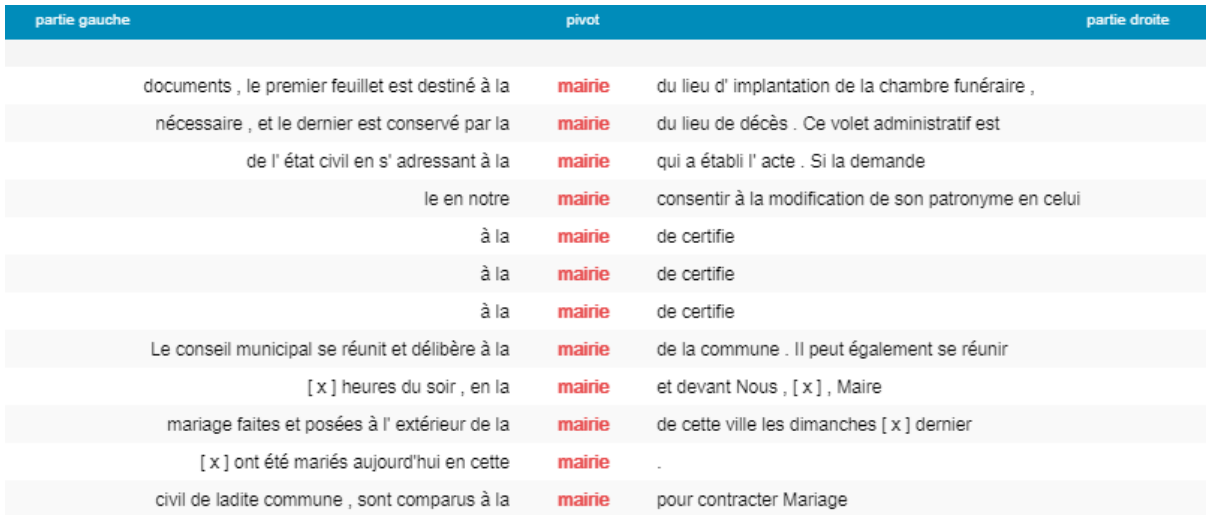

Fig. 1. Quelques concordances de mairie trouvées dans l'AMFCorpus.

La figure 1 nous montre l'élément mairie en position de noyau de quelques lignes de concordance de l'AMFCorpus générées par l'Hyperbase. Ainsi, nous avons identifié les termes désignant les lieux de célébration et d'enregistrement des mariages qui ont des attestations dans nos deux corpora diachroniques.

Ensuite, nous avons pu faire un rapport entre les occurrences des termes étudiés et les dates des documents dans lesquels ils se trouvent. Cette vérification était importante pour notre recherche car le « facteur temps » doit être pris en compte dans notre analyse.

Ainsi, nous avons observé les attestations des unités terminologiques trouvées dans le LFCorpus et l'AMFCorpus en faisant attention aux dates de délivrance et de publication de chaque sous-corpus. Pour le faire, nous y avons recherché ces termes individuellement en utilisant l'outil appelé concordance qui est disponible sur l'Hyperbase. 
Dans cette étape de notre recherche, nous avons pris en compte que « les apparitions et disparitions des attestations de certains termes au cours du temps constituent la manifestation la plus simple du changement. Elles se mesurent par une information de type présence / absence » (Tartier 2006, 348). Fondées sur cette proposition, nous avons vérifié l'évolution lexicale de la terminologie en analyse.

Après avoir identifié l'apparition et la disparition des termes analysés, nous avons recherché une bibliographie spécialisée en Histoire du Droit français et Histoire de France. Cette bibliographie a constitué le troisième corpus de notre étude et il s'appelle SupportCorpusFR ${ }^{4}$. Ce corpus nous a permis d'expliquer le comportement des termes étudiés selon les transformations socioculturelles et historiques que la France a subies au cours de la période étudiée. Dans le point suivant, nous présentons les résultats de ces analyses.

\section{L'évolution des termes étudiés}

Jusqu'en 1787, les mariages catholiques étaient considérés officiels à l'égard du Royaume français. Ainsi, l'ensemble terminologique désignant les lieux de célébration et d'enregistrement de ces mariages et de délivrance des actes de ces unions était lié au domaine religieux (catholique).

A titre d'illustration, nous présentons l'enregistrement d'une cérémonie religieuse catholique qui a eu lieu en 1757:

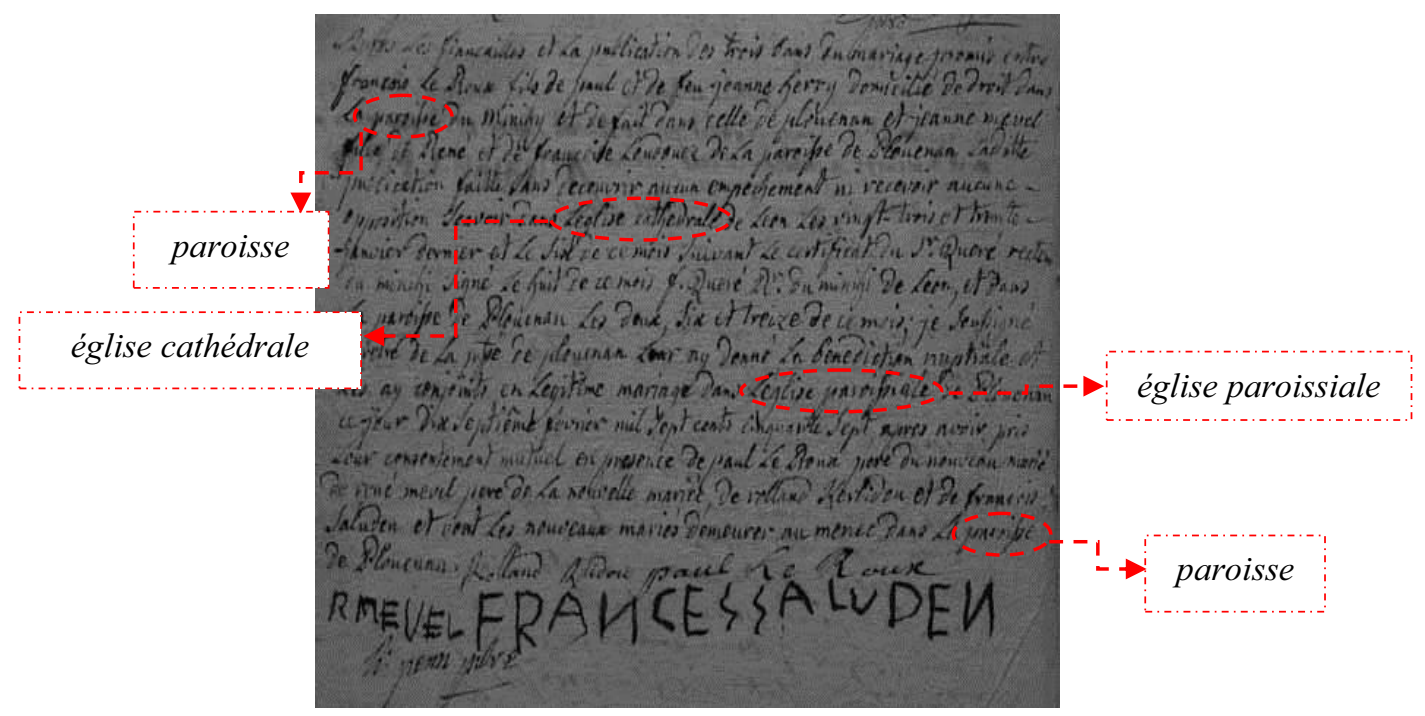

Fig. 2. Acte d'un mariage catholique célébré en 1757 (GPleyber 2015).

La figure 8 nous montre les termes paroisse, église cathédrale et église paroissiale qui font référence à l'endroit de célébration et d'enregistrement de ce mariage catholique qui a eu lieu en 1757. À cette époque-là, les mariages catholiques étaient officiellement reconnus par la Monarchie. Ainsi, pour qu'un couple français ait eu son mariage officialisé à ce moment-là, il avait dû se marier dans une Eglise catholique.

En 1539, l'Ordonnance Villers-Cotterêts édictée par le Roi a imposé aux curés l'enregistrement en français des baptêmes et des décès à des fins de contrôle royal (Millard 2011). Suite à cette détermination, l'Ordonnance de Blois en 1576 a élargi cette obligation aux mariages (Millard 2011). Ainsi, puisque les curés étaient encore officiellement responsables de l'enregistrement des baptêmes, des mariages et des décès de citoyens 
français en 1757 (l'année de délivrance de l'acte de la figure 2), l'Eglise catholique était la seule entité responsable de la célébration et de l'enregistrement des unions officiellement reconnues par le Royaume à ce moment-là.

En 1667, l'Ordonnance de Saint-Germain-en-Laye a imposé les enregistrements paroissiaux en double exemplaire. De cette façon, un acte restait dans la paroisse où il a été enregistré et l'autre était conservé dans le Greffe de la Juridiction royale de la ville (France 1667). Cette détermination monarchique a eu lieu parce que, jusqu'à cette année, l'Eglise catholique était la seule à disposer d'informations sur la naissance, le mariage et le décès de la population française, ce qui laissait l'Etat dans une situation inférieure à l'Eglise en ce qui concerne cette question une fois qu'il ne conservait pas les registres de la vie civile de la société de son royaume jusqu'alors.

Ainsi, nous considérons que les concepts designés par les termes paroisse, église cathédrale et église paroissiale, qui apparaissent dans l'acte de 1757 présenté précédemment (et dont la délivrance est, par conséquent, postérieure à ces déterminations), comprennent le trait sémantique ${ }^{5}$ endroit où ce mariage a été célébré et enregistré et où cet acte a été délivré afin de reconnaître cette union devant l'Eglise et le Royaume.

En 1787, l'Edit de Versailles a reconnu l'état civil des non-catholiques en supprimant l'exigence de leur conversion au catholicisme pour que leurs naissances, leurs mariages et leurs décès aient été enregistrés officiellement (France 1787). Selon cet Edit, « il sera au choix des parties contractantes de faire lesdites publications, ou par les curés ou vicaires des lieux où elles devront être faites, ou par les officiers de justice desdits lieux, dans la forme ci-après prescrite » (France 1787, nos soulignages). De cette façon, les citoyens français non-catholiques pouvaient choisir si leur mariage serait enregistré par les curés, qui étaient considérés des officiers civils, ou par les juges, qui jouaient le rôle d'officiers de justice. Dès lors, il était possible d'avoir un recensement de l'état civil de tous les citoyens du Royaume.

A titre d'illustration, nous présentons deux actes de mariage religieux non-catholiques délivrés en 1788, c'est-à-dire après la publication de l'Edit de Versailles (1787) :

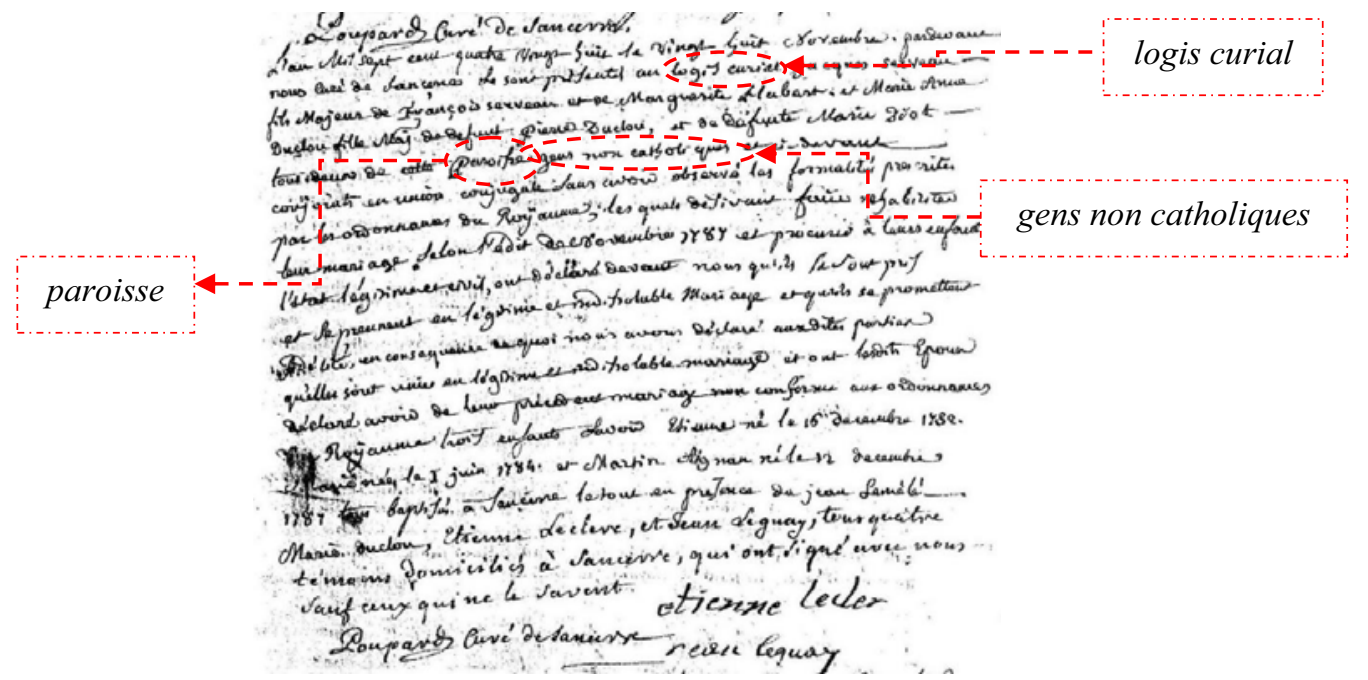

Fig. 3. Acte d'un mariage religieux non-catholique célébré en 1788 (Elodie-et-Antoine 2018). 


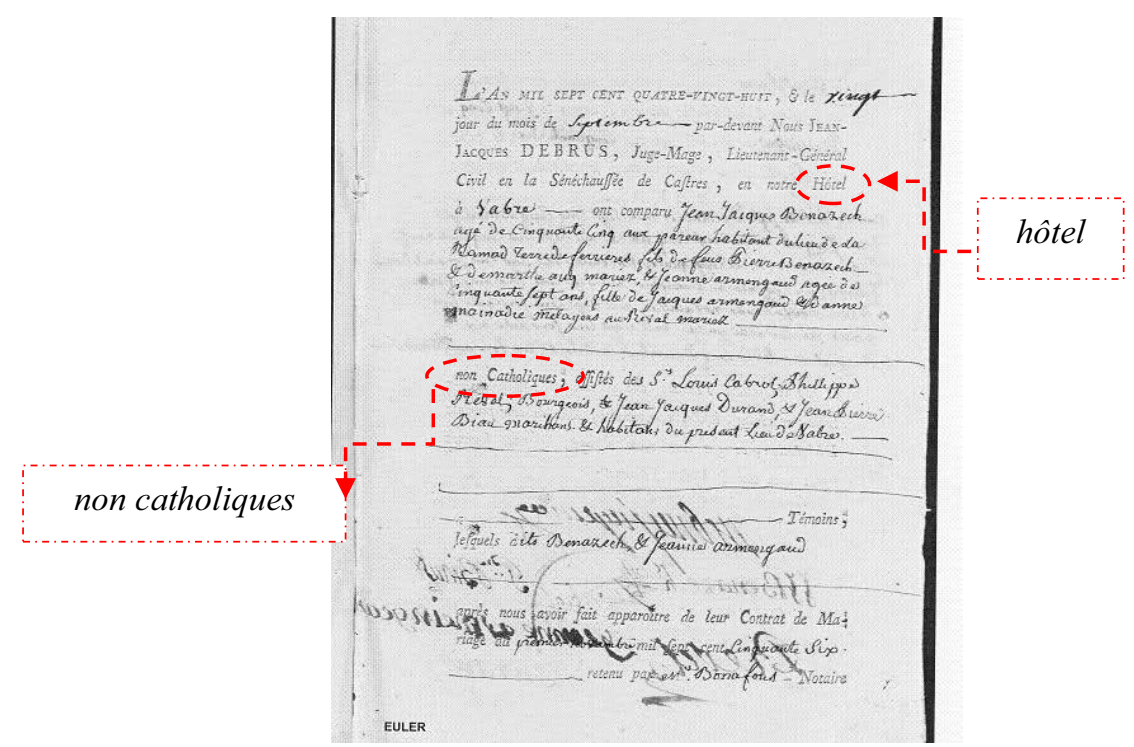

Fig. 4. Acte d'un mariage non-catholique célébré en 1788 (partie 1) (Filae 2008).

Comme nous le montre la figure 3, cet acte de mariage a été rédigé par un curé dans son logis curial, qui se trouvait dans la paroisse la plus proche d'où vivaient ces époux noncatholiques. Ainsi, nous comprenons que les termes logis curial et paroisse désignaient l'endroit où ce mariage religieux non-catholique a été officiellement célébré et enregistré et où cet acte a été délivré.

A son tour, la figure 4 nous montre un acte de mariage non-catholique enregistré par un juge royal à l'hôtel. Ainsi, dans ce cas, l'unité terminologique hôtel désigne le lieu où ce mariage a été officiellement célébré et enregistré par un juge, et où cet acte a été délivré. A cette époque-là, l'hôtel était la « résidence du roi et p. ext. de l'ensemble des personnes attachées au service du roi » (Analyse et Traitement Informatique de la Langue Française 2018). Cela peut expliquer l'apparition du terme hôtel pour nommer la maison (lieu de travail) du juge royal auquel l'Edit de Versailles a fait référence.

Fondées sur ces données, nous comprenons que les termes désignant les endroits catholiques (paroisse et église, par exemple) ont changé du point de vue sémantiqueconceptuel car, désormais, ils faisaient référence à l'un des lieux où les mariages religieux (catholiques ou non) pouvaient être célébrés et enregistrés, et les actes de ces mariages pouvaient être délivrés. D'autres lieux possibles pour l'enregistrement officiel des mariages religieux et pour la délivrance des actes prouvant ces unions étaient la maison et l'hôtel du juge, comme nous l'avons vu.

Ainsi, après la publication de l'Edit de Versailles en 1787, l'ensemble terminologique faisant référence aux endroits de célébration et d'enregistrement des mariages religieux et de la délivrance des actes de mariage était lié aux domaines religieux et juridique, puisque l'Eglise catholique et l'Etat pouvaient exercer cette fonction tous les deux.

La Révolution française (1789-1799) a rendu possible, parmi d'autres facteurs, la séparation de l'Eglise et l'Etat et l'institution du mariage civil en 1791. Dès lors, il y avait deux possibilités de mariage en France, à savoir les mariages religieux (facultatifs) et les mariages civils (officiels). L'acte de mariage civil a ensuite été créé afin d'attester du mariage civil aux yeux de l'Etat républicain.

En ce sens, le concept du terme mariage a subi un très grand changement. Par conséquent, les lieux de célébration et d'enregistrement des mariages civils et de la délivrance de leurs actes ont dû être réaménagés de manière qu'ils ont été conformes au 
nouveau concept juridique du terme mariage (civil, laïque et contrôlé uniquement par l'Etat) et à la nouvelle configuration politique et administrative de la France.

Ainsi, un nouvel ensemble terminologique qui n'est pas trouvé dans les actes de mariage religieux avant 1791 apparaît dans les actes de mariage civil exclusivement délivrés à partir de ce moment-là par l'Etat français. Ces termes ne sont plus donc associés au domaine religieux ; ils ne sont que liés au domaine juridique républicain.

A cette époque-là, les mariages civils devaient avoir lieu à la maison commune la plus proche du domicile de l'un des époux : " Art. 1er. - L'acte de mariage sera reçu dans la maison commune du lieu du domicile de l'une des parties. [...] Art. 3. - Les parties se rendront dans la salle publique de la maison commune avec quatre témoins majeurs, parents ou non parents " (France 1792 / 1989, nos soulignages). Le terme maison commune désignait donc l'endroit officiel de la célébration et de l'enregistrement des mariages civils et de la délivrance des actes de mariage. A titre d'exemple, nous présentons un acte de mariage civil délivré en 1793 :

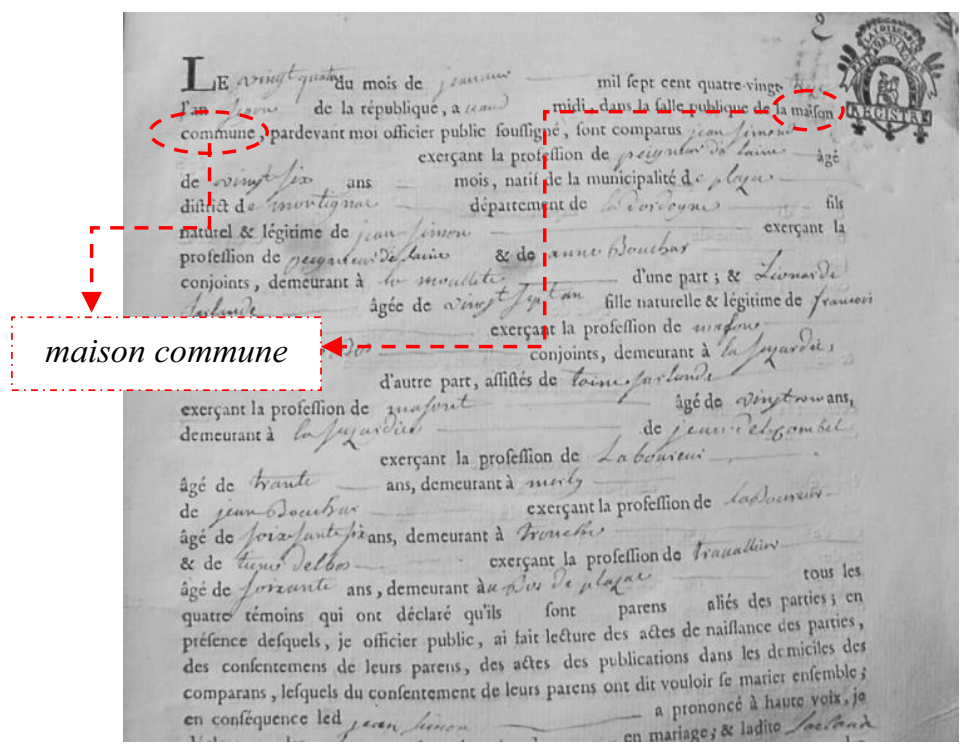

Fig. 5. Acte de mariage civil délivré en 1793 (Semond 1793).

Selon la figure 5, ce mariage civil a été célébré par un officier public dans la salle publique d'une maison commune. Par conséquent, nous comprenons que le terme maison commune désignait le lieu de la célébration et de l'enregistrement de ce mariage civil, et de la délivrance de cet acte de mariage.

Pourtant, l'observation de l'AMFCorpus nous a montré des termes différents de maison commune. Le tableau suivant rassemble quelques attestations des unités terminologiques trouvées :

Tableau 1. Transcription de quelques actes de mariage composant l'AMFCorpus.

AMF1818b : Acte de mariage de $[\mathrm{x}]$ âgée de $[\mathrm{x}]$ ans, née à $[\mathrm{x}]$ département de $[\mathrm{x}]$ du mois de $[\mathrm{x}]$ an $[\mathrm{x}]$ demeurant à $[\mathrm{x}]$ département de $[\mathrm{x}]$ fille majeure de $[\mathrm{x}]$, profession de $[\mathrm{x}]$, demeurant à $[\mathrm{x}]$, département de $[\mathrm{x}]$ et de $[\mathrm{x}] /$ et de $[\mathrm{x}]$ âgé de $[\mathrm{x}]$ ans, né à $[\mathrm{x}]$ département de $[\mathrm{x}]$ du mois de $[\mathrm{x}]$ an $[\mathrm{x}]$ profession de $[\mathrm{x}]$ demeurant à $[\mathrm{x}]$ département de $[\mathrm{x}]$ fils majeur de $[\mathrm{x}]$, profession de $[\mathrm{x}]$, demeurant à $[\mathrm{x}]$, département de $[\mathrm{x}]$ et de $[\mathrm{x}] /(\ldots)$; ont déclaré, savoir : ledit $[\mathrm{x}]$ prendre en mariage ladite $[\mathrm{x}]$ et ladite $[\mathrm{x}]$ prendre en mariage ledit $[\mathrm{x}]$; après quoi, moi, $[\mathrm{x}]$ Maire faisant les fonctions de l'officier public de l'état civil de la commune de [x] ai prononcé qu'au nom de la loi lesdits [x] sont 
unis en mariage. / De tout quoi, j'ai réglé le présent acte en la maison commune de [x] et après en avoir fait lecture aux époux, parens et témoins, je l'ai signé avec [x] ;

AMF1828 : Préfecture du Département de la [x] / EXTRAIT du Registre des Actes de Mariage / ÉTAT CIVIL (...) / Le [x] à [x] heures du matin a été prononcé à la Mairie du [x] arrondissement de [x] le Mariage de $[\mathrm{x}]$, [profession], âgé d'environ $[\mathrm{x}]$, fils majeur de $[\mathrm{x}]$ et de $[\mathrm{x}]$, tous deux décédés, [?] qu'il parait résulter de l'acte de notoriété dressé par M. [x] de [x] du Canton de [x], Département $[\mathrm{x}]$ le $[\mathrm{x}]$ homologué par jugement du Tribunal de première instance de l'arrondissement de $[\mathrm{x}]$, le $[\mathrm{x}]$ ensuite homologué par jugement du Tribunal de première instance du département de $[\mathrm{x}]$, le $[\mathrm{x}]$ le tout en forme ; ledit $[\mathrm{x}]$ demeurant à $[\mathrm{x}]$ rue de $[\mathrm{x}]$ en cet arrondissement. (...);

AMF1863 : TIMBRE IMPÉRIAL / L'an $[\mathrm{x}]$, le $[\mathrm{x}]$ à $[\mathrm{x}]$ heures du matin / Devant nous [x] Maire de la commune de $[\mathrm{x}]$, arrondissement de $[\mathrm{x}]$, département de $[\mathrm{x}]$, officier de l'état civil, ont comparu à la Mairie, / M. [x], célibataire, profession de [x], domicilié à [x] avec ses père et mère, âgé de [x] ans, né à $[\mathrm{x}]$ le $[\mathrm{x}]$, fils des époux $[\mathrm{x}]$ et $[\mathrm{x}]$ âgée de $[\mathrm{x}]$ ans et le père âgé de $[\mathrm{x}]$ ans, [profession], domiciliés à $[\mathrm{x}]$ en cette commune et ici présents et consentants. / Mlle. [x], célibataire, profession de $[\mathrm{x}]$, domiciliée $[\mathrm{x}]$ âgée de $[\mathrm{x}]$ ans, née à $[\mathrm{x}]$ le $[\mathrm{x}]$, fille des époux $[\mathrm{x}]$ et $[\mathrm{x}]$ âgés de $[\mathrm{x}]$ ans, [profession], domiciliés à $[\mathrm{x}]$ en cette commune et ici présents et consentants. (...);

AMF1904 : L'an $[\mathrm{x}]$, le $[\mathrm{x}]$ à $[\mathrm{x}]$ heures du matin devant nous, $[\mathrm{x}]$ adjoint délégué pour remplir en l'absence du titulaire les fonctions de [x] Maire et d'Officier de l'État civil de la commune du [x], arrondissement de $[\mathrm{x}]$, département des $[\mathrm{x}]$, étant en l'Hôtel-de-Ville, sont comparus : (...) (nos soulignages).

Le tableau 1 nous montre que ces mariages civils ont été célébrés dans les endroits suivants : maison commune (AMF1818b), mairie (AMF1828) et hôtel-de-ville (AMF1904). Par conséquent, nous comprenons que ces termes font référence au lieu de célébration et d'enregistrement de ces unions et de délivrance des actes qui les prouvent. Cependant, dans l'acte de 1863, mairie ne désigne que le lieu de célébration et d'enregistrement de ce mariage en tant que préfecture se réfère au lieu où cet acte a été délivré. Ces attestations peuvent être expliquées selon quelques changements législatifs par rapport à l'organisation administrative de la France qui ont eu lieu après l'institution de la République dans ce pays.

En ce sens, le décret du 14 décembre 1789 a créé les communes qui sont devenues l'une des divisions administratives françaises. Parmi d'autres aspects, ce décret a déterminé que ces communes seraient administrées par les conseillers du conseil municipal et par le maire et ses adjoints qui étaient élus par ce conseil (France 2018). Ces deux institutions administratives se situaient dans la maison commune (Nogentrev 2016). Ainsi, le concept de maison commune comprenait le trait sémantique l'endroit où se trouvaient la mairie et le conseil municipal.

Comme nous l'avons vu précédemment, la Constitution de 1791 a déterminé que la maison commune serait le lieu officiel de célébration et d'enregistrement des mariages civils et de délivrance des actes de mariage. Pour s'occuper de ce poste dans cet endroit, l'une ou plusieurs personnes parmi les membres du conseil municipal seraient nommées (France 1792 / 1989). Ainsi, il n'y avait aucun fonctionnaire spécifique qui s'occupait de cette fonction, puisque la détermination de cette charge dépendait du vote du conseil. En conséquence de cela, il n'existait pas encore un secteur spécifique au sein de la maison commune chargé de cette fonction.

Ce scénario a changé après la loi du 17 février 1800. Cette loi a été fondamentale pour l'organisation politique et administrative de la France, ainsi que du reste de l'Occident, car elle a été la base pour la formation du Droit administratif occidental (Mafra 2005). L'une de ses déterminations a établit que les maires et leurs adjoints seraient désormais les responsables de la célébration des mariages, de l'enregistrement de l'état civil et de la délivrance des actes (France 1800 / 2000). A partir de ce moment-là, la mairie est donc devenue l'endroit officiel où ces agents exerçaient leurs fonctions en tant qu'officiers d'état 
civil. Ainsi, ce changement explique les attestations du terme mairie dans les actes de mariage qui ont été délivrés après 1800 et qui composent notre corpus.

Par rapport aux grandes communes (Paris, Lyon et Marseille, par exemple), où il y avait plus d'une mairie, le décret de 1789 a déterminé que l'hôtel de ville serait le siège du conseil municipal et de la mairie principale de ces communes (Serieys 2019). Ainsi, le terme hôtel de ville désignait le lieu où se trouvaient ces deux établissements publics dont les fonctionnaires dirigeaient une grande commune. Dans ces villes, il y avait une mairie pour chaque arrondissement.

De cette façon, nous comprenons que, dans trois cas du tableau 1 (à savoir AMF1818b, AMF1828 et AMF1904), il y a d'autres caractéristiques de la configuration conceptuelle de ces termes qui sont similaires entre eux, outre le trait sémantique lié au lieu de célébration e d'enregistrement de ces mariages. Pourtant, il y a quelques différences entre ces unités terminologiques qui tiennent surtout au fait que l'hôtel de ville désignait spécifiquement la mairie centrale d'une grande commune ; à son tour, mairie faisait référence aux autres mairies d'une grande commune et la seule mairie d'une commune plus petite ; et enfin, maison commune désignait un concept plus générique que ceux des deux autres termes, car il comprenait, dans sa configuration sémantique, le trait bâtiment public dans lequel se trouvaient le conseil municipal et la mairie d'une commune.

Entre ces termes et préfecture, les différences sont encore plus remarquables. En ce sens, la loi du 17 février 1800, que nous avons déjà mentionnée, en plus de clarifier les devoirs des fonctionnaires français, a établit notamment le suivant : « il y aura dans chaque département un préfet, un conseil de préfecture, et un conseil général de département qui rempliront les fonctions exercées maintenant par les administrations et commissaires de département 》 (France 1800 / 2000). Ainsi, la préfecture a été créée pour être chargée de l'administration des départements en France. Ce terme ne comprenait pas donc le trait sémantique lieu où les services municipaux sont faits et, à cause de cela, il était différent du point de vue sémantique et conceptuel des termes maison commune, mairie et hôtel de ville.

Cependant, dans le tableau 1, nous voyons que l'unité terminologique préfecture fait référence au lieu où l'extrait de l'acte de mariage de 1828 a été délivré, mais non au lieu où ce mariage a été célébré et enregistré. Cette attestation peut être expliquée selon les lois du 20 septembre 1792 et du 17 février 1800. La première loi a déterminé que les actes d'état civil devraient être enregistrés en double exemplaire. Ainsi, une copie de ces documents devait être envoyée aux archives des directoires des départements où elle serait conservée, et l'autre se trouvait où leurs enregistrements ont été effectués (France 1792). La deuxième loi a établit que les directoires de 1792 seraient remplacés par les préfectures (France 1800 / 2000). De cette façon, une copie de l'état civil devait être envoyée à ces institutions à partir de ce moment-là.

En possession de ces copies, les préfectures pouvaient délivrer des extraits des actes d'état civil. Pour cette raison, la configuration conceptuelle du terme préfecture comprenait le trait sémantique l'un des endroits où la copie des actes de naissance, de mariage et de décès étaient déposée et où l'extrait de ces documents pouvait être délivré. Dans notre corpus, cette unité terminologique est utilisée dans ce sens, puisqu'elle figure dans un acte de mariage daté de 1828 (c'est-à dire après la publication de la loi de 1800).

Ainsi, le concept désigné par le terme mairie, dont l'attestation peut être également observée dans l'acte de 1828, ne comprend pas le trait sémantique lieu de délivrance de cet extrait. Dans ce cas, cette unité terminologique se réfère seulement au lieu de célébration et d'enregistrement de ce mariage civil.

Bien que la loi française ait changé au fil du temps, de 1800 à nos jours il n’y a eu aucun changement concernant l'endroit officiel de la célébration et de l'enregistrement des mariages civils et de la délivrance de leurs actes. Nous pouvons faire cette affirmation selon nos analyses de l'ensemble terminologique trouvé dans l'AMFCorpus. Pour illustrer les 
attestations analysées, le tableau 2 montre la transcription partielle des actes de mariage délivrés en 1923, 1988 et 2005 respectivement :

Tableau 2. Transcription de quelques actes de mariage civil de l'AMFCorpus.

AMF1923 : MAIRIE DE $[\mathrm{x}]$ / EXTRAIT des minutes des actes de mariage / arrondissement de $[\mathrm{x}] /$ a été célébré le mariage de $[\mathrm{x}]$ né à $[\mathrm{x}]$ / née à $[\mathrm{x}]$ / un contrat de mariage a été reçu $[\mathrm{x}]$ notaire / Certifie le présent extrait conforme aux indications portées au registre par nous, officier d'état civil délégué par le Maire du 17e arrondissement ;

AMF1988 : MAIRIE DE [x] / Acte de mariage - Copie Intégrale / Acte de mariage $\mathrm{n}^{\mathrm{o}}[\mathrm{x}] /$ devant Nous, ont comparu publiquemente en la Maison Commune / [x] âgé de $[\mathrm{x}]$ / [x] âgée de [x] / Sur notre interpellation, les futurs époux ont déclaré qu'il n'a pas été fait de contrat de mariage (...);

AMF2005 : (...) devant nous ont comparu publiquement en la maison commune / Epoux / Nom [x] / Prénoms $[\mathrm{x}]$ / Né à $[\mathrm{x}] /$ Fils de $[\mathrm{x}]$ / et de $[\mathrm{x}] /$ Epouse / Nom $[\mathrm{x}]$ / Prénoms $[\mathrm{x}]$ / Née à $[\mathrm{x}]$ / Fille de $[\mathrm{x}] /$ et de $[\mathrm{x}](\ldots)$ (nos soulignages).

Le tableau 2 nous montre que les termes maison commune et mairie ont été utilisés pour désigner les lieux où ces mariages civils ont été célébrés et enregistrés et où ces actes ont été délivrés. Ainsi, les attestations de ces unités terminologiques dans les documents AMF1923, AMF1988 et AMF2005 nous confirment que leur configuration sémantique n'a pas changé après la loi du 17 février 1800 en ce qui concerne cette question spécifique.

En 2017, le décret $n^{\circ} 2017-270$ a permis la célébration des mariages civils dans un autre bâtiment public choisi par le maire (France 2017a). Cette possibilité a été revendiquée par les maires car certaines mairies disposaient de très petites salles où la célébration d'un mariage civil ne pouvait être bien faite (France 2017b).

En conséquence de cela, les actes de mariage civil délivrés après ce décret pourront faire référence à des endroits différents de la mairie. Ce sera où la célébration de ces mariages ${ }^{6}$ aura lieu. De cette façon, nous comprenons que d'autres termes pourront apparaître dans ces documents pour désigner ces endroits.

\section{Synthèse et quelques considérations}

En définitive, il est possible de constater qu'au fil du temps, il y a eu des changements en France concernant les institutions chargées de la célébration et de l'enregistrement des mariages officiels, ainsi que de la délivrance des actes qui les prouvent auprès de l'Etat et de la société. Par conséquent, la terminologie désignant ces lieux a accompagné ces transformations.

En ce sens, les termes relatifs aux endroits religieux sont trouvés dans les actes des mariages célébrés jusqu'en 1787 pour indiquer ces lieux. Entre 1787 et 1791, on découvre des termes religieux et des termes en rapport avec l'administration publique du Royaume dans les actes attestant l'état civil des citoyens français à cette époque-là. Ainsi, les termes église et maison du juge, par exemple, pouvaient apparaître dans les actes de mariage selon le lieu d'enregistrement de ces unions et de délivrance de leurs respectifs actes.

A la suite de l'institution du mariage civil en 1791, l'unité terminologique maison commune désignait les endroits où ont eu lieu les célébrations et les enregistrements des mariages civils et la délivrance des actes qui servaient de preuve. De 1800 à nos jours, ces lieux sont surtout désignés par les termes maison commune, mairie et hôtel de ville.

De cette façon, nous constatons que les termes qui désignent ces lieux dans notre champ d'étude ont accompagné les transformations socioculturelles et politiques que la France a subies au cours des siècles. En conduisant cette étude, nous pouvons observer le rapport 
entre ces termes et les questions religieuses, ainsi que le changement de conception politique après l'établissement de la République dans ce pays.

Outre les questions liées à ces aspects socioculturels et historiques, il est intéressant de souligner la dynamique de la production lexicale de cette terminologie dans le domaine du Droit civil. En ce sens, nous constatons que les termes désignant les lieux liés à l'institution de l'Eglise catholique ont commencé à être utilisés dans le cadre juridique après les Ordonnances Villers-Cotterêts (1539) et de Blois (1576). Cet ensemble terminologique a continué à être utilisé pour faire référence aux lieux de célébration et d'enregistrement des mariages officiellement reconnus jusqu'en 1787, lorsque 1'Edit de Versailles a introduit une terminologie en lien avec l'administration juridique du Royaume pour désigner certains de ces endroits. Ainsi, des termes religieux et des termes juridiques ont commencé à coexister dans le domaine en question.

Après l'institution du mariage civil et laïque et et l'absence d'influence de l'Eglise dans les affaires de l'Etat républicain, la terminologie liée à l'institution catholique est devenue obsolète en Droit civil, c'est-à-dire que ces termes sont devenus des nécrologismes dans ce domaine de spécialité, bien qu'ils aient continué à être employés en Droit canonique. A son tour, une nouvelle terminologie a été créée afin de souligner, dans son expression, la distance entre le régime précédent (monarchique) et le régime révolutionnaire (républicain). Ces termes qui étaient, tout d'abord, néologiques sont devenus stables au cours du temps et continuent à être utilisés de nos jours dans le domaine étudié.

Nous remercions la Fondation de Soutien à la Recherche de l'Etat de São Paulo (FAPESP) pour son soutien financier.

\section{Références bibliographiques}

Alves, I. M. (2003). Socioterminologie. Une approche sociolinguistique de la Terminologie. Tradterm, 9, 229-32.

Alves, I. M. (2007). Neologismo. Criação lexical. São Paulo : Ática.

Alves, I. M. (2009). Neologia e implicações textuais. Anais do VI Congresso Internacional da ABRALIN, 6, 1821-1825.

Angers. (2019). Se marier à Angers. Disponible sur : http://www.angers.fr/servicesdemarches/demarches-pour-les-particuliers/etat-civil/se-marier-a-angers/index.html. Consulté le 4 août 2019.

Analyse et Traitement Informatique de la Langue Française (ATILF). (2019). Le Trésor de la Langue Française Informatisé. Disponible sur : http://atilf.atilf.fr/. Consulté le 15 mai 2019.

Barros, L. A. (1998). Aspects de la productivité lexicale dans le domaine des espaces protégés du Brésil : la variation orthographique, morphologique et syntaxique. Acta Semiotica et Linguistica, 7, 45-86.

Barros, L. A. (2004). Curso Básico de Terminologia. São Paulo : Editora da Universidade de São Paulo.

Bortolato, C. P. (2013). Uma proposta de tradução da terminologia jurídica do Ancien Régime presente na peça Les Plaideurs de Jean Racine. Thèse de master soutenue dans l'Université de São Paulo (USP), à São Paulo, Brésil. Disponible sur : http://www.teses.usp.br/teses/disponiveis/8/8146/tde-18112013131108/publico/2013_CarolinaPoppiBortolato_VCorr.pdf. Consulté le 27 avril 2016.

Cabré, M. T. (1998). La Terminologie: théorie, méthodes et applications. Traduit, adapté et mis à jour par Monique Cormier et John Humbley. Ottawa : Presses de l'Université d'Ottawa; Paris : Armand Colin. 
Cabré, M. T. (1999). La terminología: representación y comunicación: elementos para una teoría de base comunicativa y otros artículos. Barcelona : IULA.

Conceição, M. C. (1999). Terminologie et transmission du savoir: (re)construction(s) de concepts. In : Delavigne, V.; Bouveret, M. (Eds.). Sémantique des termes spécialisés. Rouen : Presses Universitaires de Rouen, 33-42.

Curti-Contessoto, B. (2018). Terms designating places for the celebration and civil registration of marriages and places for the issuance of marriage certificates in Brazilian history. Terminàlia Revista Semestral de la Societat Catalana de Terminologia, 18, 14-24.

Curti-Contessoto, B. F. (2019). Terminologia de certidões de casamento: estudo terminológico bilíngue e elaboração de glossário português-francês. Thèse de doctorat soutenue à l'Université de l'Etat de São Paulo, Campus de São José do Rio Preto, Brésil. Disponible sur : https://repositorio.unesp.br/handle/11449/183611. Consulté le 24 avril 2020.

Dalloz, V. A. D. \& Dalloz, A. (1845). Répertoire méthodique et alphabétique de législation, de doctrine et de jurisprudence en matière de droit civil, commercial, criminel, administratif, de droit des gens et de droit public. 2. Disponible sur : https://play.google.com/books/reader?id=Zk0DAAAAQAAJ\&printsec=frontcover\&output=reade r\&hl=pt_BR\&pg=GBS.PA490. Consulté le 12/09/2015.

Depecker, L. (2002). Entre signe et concept. Eléments de terminologie générale. Paris : Presses Sorbonne Nouvelle.

Dury, P. \& Drouin, P. (2011). When Terms Disappear from a Specialized Lexicon: A Semi-automatic Investigation into Necrology. ICAME Journal, 35, 19-33.

Dury, P. (2013). Que montre l'étude de la variation d'une terminologie dans le temps. Quelques pistes de réflexion appliquées au domaine médical. Debate Terminológico, 9, 2-10.

Elodie-et-Antoine. (2018). Mariage de Jaques SERVEAU et Marie DUCLOU - Archives du Cher$3 E$ 1036. Disponible sur : http://www.elodie-et-antoine.fr/ra/?tag=protestants. Consulté le 18 décembre 2018.

Filae. (2008). Ancêtres protestants : liste de liens en première page. 2008. Disponible sur : https://www.filae.com/v4/forums/recherches-genealogiques-ancetres-protestants-liste-de-liens-enpremiere-page-t1149677-p1.html. Consulté le 18 décembre 2018.

France. (1667). Ordonnance de Louis XIV... donnée à Saint Germain en Laye au mois d'avril 1667. Disponible $\quad$ sur $\quad$ : http://numelyo.bmlyon.fr/f_view/BML:BML_00GOO0100137001101319999/IMG00000022. Consulté le 19 décembre 2018.

France. (1787). Edit de Versailles (7 novembre 1787). Disponible sur : http://huguenotsweb.free.fr/histoire/edit1787.htm. Consulté le 18 décembre 2018.

France. (1791). Constitution de 1791. Disponible sur : http://www.conseil-constitutionnel.fr/conseilconstitutionnel/root/bank/pdf/conseil-constitutionnel-5082.pdf. Consulté le 10 juin 2015.

France. (1792/1989). Loi du 20 Septembre 1792 sur les causes, le mode et les effets du divorce. Population \& Société, $240 . \quad$ Disponible $\quad$ sur https://www.ined.fr/fichier/s_rubrique/18939/pop_et_soc_francais_240.fr.pdf. Consulté le 21 novembre 2018.

France. (1800/2000). Loi du 28 Pluviôse An VIII (17 février 1800). Disponible sur : https:/www.upicardie.fr/curapp-revues/root/45/Annexe.pdf_4a0d2b2344d22/Annexe.pdf. Consulté le : 15 décembre 2018.

France. (2017a). Décret $n^{\circ}$ 2017-270 du ler mars 2017 relatif à la délégation des fonctions d'officier de l'état civil exercées par le maire et au lieu de célébration des mariages. Disponible sur : https://www.legifrance.gouv.fr/affichTexte.do?cidTexte=JORFTEXT000034128626\&categorieLi en=id. Consulté le 4 août 2019. 
France. (2017b). Loi J21 : un décret autorise la célébration du mariage hors de la mairie et précise le champ de la délégation des fonctions d'officier d'état civil. Disponible sur : https://etatcivil.legibase.fr/actualites/veille-juridique/loi-j21-un-decret-autorise-la-celebration-du-71476/.

Consulté le 4 août 2019.

France. (2018). La commune. Disponible sur : https://www.collectivites-locales.gouv.fr/commune. Consulté le 8 juillet 2019.

Freixa, J. (2006). Causes of denominative variation in terminology: A typology proposal. Terminology, 12, 51-78.

Freixa, J. (2014). La variación denominativa en terminología: tipos y causas. In : Isquerdo, A. N. \& Dal Corno, G. O. M. (Eds.). As Ciências do Léxico: Lexicologia, Lexicografia e Terminologia. Campo Grande : Ed. UFMS, 311-329.

Gaudin, F. (2005). La socioterminologie. Langages, 157, 80-92. Disponible sur : https://doi.org/10.3406/lgge.2005.976. Consulté le 23 avril 2020.

GPleyber. Site spécialisé en documents anciens. Disponible sur : http://gpleyber.free.fr/actes\%20anciens.html. Consulté le 25 juin 2015.

Krieger, M. G. \& Finatto, M. J. B. (2004). Introdução à Terminologia: teoria e prática. São Paulo : Contexto.

Mafra, F. (2005). Primeiras noções de Direito Administrativo. Âmbito Jurídico, 23.

Millard, E. (2011). Le rôle de l'état civil dans la construction de l'Etat. In : Blanc, F.-P. Mélanges en l'honneur du Doyen. Toulouse : Presses Universitaires de Perpignan et Presses Universitaires de Toulouse, 721-727.

Nogentrev. (2016). Les municipalités. Nogent-le-Rotrou sous la Révolution française. Disponible sur : http://www.nogentrev.fr/archives/2016/03/05/33469324.html. Consulté le 8 juillet 2019.

Pavel, S. \& Nolet, D. (2003). Manuel de Terminologie. Gatineau : Quebec.

Picton, A. (2014). The dynamics of terminology in short-term diachrony: A proposal for a corpusbased methodology to observe knowledge evolution. In : Temmerman, R. \& Van Campenhoudt, M. (Eds). Dynamics and Terminology: An interdisciplinary perspective on monolingual and multilingual culture-bound communication. Amsterdam : John Benjamins Publishing Company, 160-182.

Semond. (1793). Acte de mariage de Jean SEMOND et Leonarde SARLANDE (24/01/1793). Disponible sur: http://semondfamily.pagesperso-orange.fr/11040048.JPG. Consulté le 15 décembre 2018.

Serieys, J. (2019). 14 décembre 1789 : Création des communes par la Révolution française. Disponible sur : http://www.gauchemip.org/spip.php?article10803. Consulté le 8 juillet 2019.

Simonis, S. \& Simonis, G. (Eds.). (2001). El amor y el matrimonio. Santafé de Bogotá : San Pablo.

Tartier, A. (2006). Analyse automatique de l'évolution terminologique. Actes du Traitement Automatique des Langues Naturelles (TALN). Disponible sur : http://www.revuei3.org/hors_serie/annee2006/revue_33_hs2006_01_05.pdf. Consulté le 27 avril 2016.

\section{Notes :}

1 Il est important de souligner qu'il $y$ a une fine ligne qui distingue unité terminologique d'unité lexicale, surtout en ce qui concerne les études terminologiques 
dévelopées selon un point de vue diachronique (cf. Dury 2013). Cependant, dans cet article, nous ne discutons pas le fond de ce sujet.

2 Tous les actes de mariage qui composent l'AMFCorpus ont été acquis sur Internet. Pour cette raison, nous comprenons qu'ils sont du domaine public et que nous pouvons en inclure certains dans cet article à titre d'illustration de nos analyses.

${ }^{3}$ Disponible sur http://hyperbase.unice.fr/.

${ }^{4}$ La liste complète de tous les documents légaux qui composent le LFCorpus et de la bibliographie rassemblée dans le SupportCorpusFR peut être trouvée dans notre thèse de doctorat (cf. Curti-Contessoto 2019).

5 Dans ce travail, nous considérons que, selon un point de vue linguistique, les caractéristiques légistatives liées aux lieux de célébration et d'enregistrement des mariages officiels français et de délivrance des actes consistent en traits sémantiques composant la configuration conceptuelle des termes étudiés.

${ }^{6}$ A titre d'exemple, il y a, sur le site de la mairie d'Angers, une publication qui se lit comme le suit : " Vous vous mariez à Angers un samedi entre le 6 avril 2019 et le 31 décembre 2019 ? Votre cérémonie se déroulera exceptionnellement à l'Hôtel de Livois" (Angers 2019). Ce changement de lieu, dans ce cas, est arrivé à cause des travaux faits dans cette mairie. Dans les actes de mariage civil y délivrés pendant cette période, on trouvera, certes, l'expression Hôtel de Livois nommant le lieu de célébration de ces mariages. 